\title{
Médiévales
}

Langues, Textes, Histoire

62 | printemps 2012

Hagiographie et réforme dans l'Occident latin

\section{Scott G. Bruce, Silence and Sign Language in Medieval Monasticism. The Cluniac Tradition (ca 900-1200)}

Cambridge, Cambridge University Press, 2009 [2007], 209 p. (Cambridge Studies in Medieval Life and Thought, Fourth Series, 68)

\section{Gabriel de Carvalho Godoy Castanho}

\section{(2) OpenEdition}

Journals

Édition électronique

URL : https://journals.openedition.org/medievales/6695

DOI : $10.4000 /$ medievales. 6695

ISSN : $1777-5892$

Éditeur

Presses universitaires de Vincennes

Édition imprimée

Date de publication : 2 juin 2012

Pagination : 189-191

ISBN : 978-2-84292-346-4

ISSN : 0751-2708

Référence électronique

Gabriel de Carvalho Godoy Castanho, « Scott G. Bruce, Silence and Sign Language in Medieval

Monasticism. The Cluniac Tradition (ca 900-1200)», Médiévales [En ligne], 62 I printemps 2012, mis en

ligne le 29 juin 2012, consulté le 24 avril 2022. URL : http://journals.openedition.org/medievales/6695 ; DOI : https://doi.org/10.4000/medievales.6695

Ce document a été généré automatiquement le 24 avril 2022

Tous droits réservés 


\title{
Scott G. Bruce, Silence and Sign Language in Medieval Monasticism. The Cluniac Tradition (ca 900-1200)
}

Cambridge, Cambridge University Press, 2009 [2007], 209 p. (Cambridge

Studies in Medieval Life and Thought, Fourth Series, 68)

\author{
Gabriel de Carvalho Godoy Castanho
}

1 Les anges vivent dans le silence et la psalmodie; les hommes dans la confusion des langues. Cette idée-force, si chère aux ascètes chrétiens des premiers siècles qui ont abandonné le bruit des villes à la recherche des milieux peu peuplés, est au centre de l'argumentation du beau livre de Scott G. Bruce. Entièrement basé sur une documentation écrite - avant tout normative, et particulièrement coutumière --, le livre est bien cadré dans le champ analytique de l'idéation, à savoir le rôle de l'analogie " moine-ange » dans la normalisation de la communication quotidienne des moines. Si nous pouvons le croire présent depuis les débuts du monachisme, l'idéal d'abandon de la parole n'était pas homogène, comme l'auteur le démontre bien. Pendant des siècles, ce renoncement a été au centre des polémiques monastiques, devenant largement accepté au XII ${ }^{e}$ siècle seulement, après le tournant fondamental orchestré à Cluny.

2 En opposition à la tradition classique qui associe le beau parler à l'activité politique citoyenne, le monachisme de l'Antiquité tardive valorise le silence en tant qu'exercice spirituel ascétique et discipline sociale. Scott G. Bruce soutient que cette ligne de conduite a eu une double fonction: éviter de laisser le moine être séduit par les aménités d'une rhétorique peccamineuse, ainsi que favoriser l'humilité et l'obéissance, aspects primordiaux de la société monastique. Cela ne signifie pas que les religieux de l'Antiquité tardive ont abandonné la parole, mais plutôt que l'idéal de silence jouait un rôle de grande importance dans l'organisation sociale par le biais du contrôle des formes de communication au sein de la communauté. Le désir du silence à l'époque carolingienne a surtout encouragé l'état révérenciel des moines et la distinction entre la quiétude du cloître et le bruit du monde. Finalement, le $x^{e}$ siècle, au-delà de la réaffirmation des topiques de l'humilité et de l'obéissance, attribue à la métaphore 
moine-ange un aspect apocalyptique de première importance: les moines de Cluny essayent de reproduire sur terre le silence éternel des êtres supérieurs qui accompagnera la résurrection du Christ à la fin du monde. Ce changement de perspective a trouvé des résistances et a pris à Cluny un aspect particulier chez les ascètes de l'époque.

3 Parmi les trois qualités angéliques fondamentales (pureté sexuelle, psalmodie et silence), les clunisiens sont connus par leur effort d'émulation des éléments sonores, qui les amène à concevoir le silence comme une vertu en soi-même. Il s'agit d'une " nouvelle idéologie de l'ascétisme chrétien qui associe la glorification du silence à l'idéal d'une vie angélique accomplie dans un corps mortel». C'est bien là que se trouve le fondement de leur prestige auprès des laïcs et d'autres membres de l'Église : l'efficacité de leurs oraisons est assurée par la diffusion de l'acceptation de l'idéologie à la base de leur fonction dans la société. Le silence est ainsi un créateur de distinction sociale, fait fort intéressant sous-jacent au plan argumentatif du livre, mais non exploité par l'auteur dans sa dimension d'élément structurant de l'institution ecclésiale.

4 D'une écriture très agréable rendant sa lecture accessible à tous les publics, le livre est divisé en cinq chapitres. Le premier («Uttering No Human Sound ») aborde les réussites et les limites de la discipline du silence chez les moines de Cluny vis-à-vis de la logique du rapport moine-ange. En partant de l'acte de donation de Guillaume III, duc d'Aquitaine, des textes d'Odon, deuxième abbé de Cluny (927-942), ainsi que de la vita Odonis rédigée par Jean de Salerne, Scott G. Bruce met au premier plan l'emploi du vocable coelestis comme qualificatif de la vie menée par les moines de Cluny. Selon l'auteur, l'exhortation contre le discours mondain et en faveur de son contrôle est une des principales marques de l'abbatiat d'Odon. De même, l'approche, établie par Jean de Salerne, entre les clunisiens et la tradition du silence sacré, diffusé par la Bible et par les premiers ermites chrétiens, permet à l'auteur de visiter la topique de l'isolement monastique. Cette perspective n'empêche pas son contraire. En effet, la place privilégiée donnée au silence à Cluny, selon Jean de Salerne, attirait la critique des contemporains qui la comprenaient comme une création nouvelle. Il faudra attendre deux siècles pour que cette méfiance se transforme en éloge, après l'établissement d'un langage de signes bien cadré.

5 Le chapitre suivant ("The Training of the Hand») présente le lexique des signes pratiqué à Cluny au $\mathrm{XI}^{\mathrm{e}}$ siècle afin de démontrer son aspect linguistique. Le langage de signes créé à Cluny vise les activités quotidiennes élémentaires comme l'alimentation et la correction des mœurs, notamment ceux des novices. Basé sur un répertoire de noms sans rapport avec des structures complexes, il s'agit d'un langage volontairement simple, et non d'un système trop proche du langage parlé qui pourrait faciliter une communication de type mondain et, par conséquent, mettre en cause le silence luimême. Ensuite ("A Silent Commerce of Signs»), nous passons à une enquête sur les fonctions et l'application des signes dans les coutumes de Bernard de Cluny et d'Ulrich de Zell (fin $\mathrm{XI}^{\mathrm{e}}$ siècle). Documentation à l'appui, l'auteur soutient que l'apparition et l'importance du langage des signes à Cluny au xi ${ }^{\mathrm{e}}$ siècle sont une solution efficace dans un milieu plurilinguistique où les accents et les connaissances du latin poseraient des difficultés à la communication quotidienne. Le cas d'Orderic Vital est cité afin de démontrer comment les différents accents du latin médiéval sont créateurs de difficultés de communication et des sentiments d'identité. 
6 Dans l'étape suivante («Transmission and Adaptation»), l'argumentation trace l'expansion et les modifications des signes monastiques au-delà de la Bourgogne. L'auteur vise à établir l'influence de Cluny dans le contenu des trois autres codes de gestes produits avant le XII ${ }^{\mathrm{e}}$ siècle, à Fleury, à Canterbury et à Hirsau. Les manuscrits rédigés dans ces maisons ne présentent pas des principes de langage au-delà de la structure sémiotique clunisienne et, par conséquent, ne dépassent pas les fonctions de la communication silencieuse dans l'abbaye. Depuis le début du $\mathrm{XI}^{\mathrm{e}}$ siècle, Fleury possède un lexique propre, de base clunisienne, mais adapté à la coutume locale. Canterbury, selon l'auteur, aurait organisé son système autour de l'an mil, après la visite d'un moine de Fleury, maison réformée par Odon de Cluny vers 930. Là aussi, le langage a maintenu la simplicité tout en étant enrichi par le vocabulaire local. Finalement, S.G. Bruce soutient que le fort aspect liturgique du lexique composé dans les années 1080 à Hirsau ne s'oppose pas au caractère didactique de la liste de Cluny. Bien au contraire. Les deux catalogues peuvent être compris comme étant complémentaires, si l'on pense que les moines de la Forêt Noire ont, peut-être, tout simplement mis par écrit ce qui était pratiqué, mais non rédigé, à Cluny - à cause de la fonction didactique-moralisante du langage clunisien.

7 Le dernier chapitre («Continuity and Criticism ») offre une analyse de l'expansion et de l'adaptation des signes au XII ${ }^{\mathrm{e}}$ siècle. L'auteur aborde l'idéal du silence et la pratique des signes chez les cisterciens (continuité de la valorisation du silence et des gestes), les chanoines réguliers (valorisation des gestes et critique du silence) et la Grande Chartreuse (valorisation du silence, critique des gestes). À cause des différentes formes d'attachement à la cura animarum, clunisiens, cisterciens, chanoines et chartreux possédaient des niveaux distincts d'acceptation $\mathrm{du}$ silence, tout en gardant la reconnaissance de sa valeur. Les cisterciens et les chanoines réguliers ont adopté le langage des signes à partir $\mathrm{du} \mathrm{xII}^{\mathrm{e}}$ siècle, sous influence du modèle clunisien de simplicité lexicale. Au-delà des dossiers mentionnés, l'auteur cite le cas d'Hildegarde de Bingen et de la documentation conciliaire du $\mathrm{XII}^{\mathrm{e}}$ siècle pour conclure que la valeur positive du silence et du langage des signes était une des caractéristiques fondamentales du monachisme de l'époque, définissant leur identité dans un moment de croissante diversité religieuse.

8 Finalement, la section conclusive du livre présente un bon résumé de chaque étape argumentative et ouvre les portes de la modernité en proposant l'hypothèse de l'origine médiévale (bénédictine) de l'éducation des enfants sourds dans l'Espagne du $\mathrm{XVI}^{\mathrm{e}}$ siècle. À travers le cas du moine Pedro Ponce qui a fait parler à haute voix deux enfants sourds, Scott G. Bruce évoque l'exclusion sociale des muets afin de mettre en évidence les rapports entre le rationnel du silence et des signes monastiques organisés dès le $\mathrm{x}^{\mathrm{e}}$ siècle et les réussites de l'éducation des enfants sourds dans des abbayes espagnoles du XVI ${ }^{\mathrm{e}}$ siècle.

9 L'auteur a réussi à réunir les éléments principaux de la mentalité du silence et de la communication gestuelle des moines médiévaux dans un livre court avec une écriture claire, agréable et accessible à tous les publics. Bien qu'ayant passé trop brièvement sur quelques-unes des problématiques les plus épineuses de son propos, notamment les théories du langage des signes et les limites des données issues d'une documentation coutumière, l'ouvrage est une introduction solide pour tous ceux qui veulent comprendre les traces principales de la pensée ascétique autour du silence dans la tradition monastique chrétienne. De plus, Scott G. Bruce présente quelques réflexions 
particulièrement intéressantes et productives, comme la démarche d'analyse croisée des coutumiers clunisiens de Bernard et d'Ulrich avec le lexique de signes qui existait à la même époque. Elle lui permet d'affirmer le caractère fondamental de la communication dans le silence: encourager les moines «à interagir dans ce qui relève de la pratique mondaine sans avoir recours à des paroles ou des à signes, imposant ainsi une limite aux lieux où le langage des signes était permissif. [...] Par ailleurs, il est évident que les novices ont appris ce langage silencieux surtout pour reconnaître les signes d'instruction et de réprimande employés par leur maître pendant leur noviciat» (p.172). On voit bien que le silence ascétique est une forme de pratique de l'obéissance et de l'humilité. Cependant, nous pourrions ajouter que cette éthique du silence fait partie d'une certaine utopie de l'isolement, horizon toujours présent dans le monachisme chrétien, et qui, aux XI $\mathrm{I}^{\mathrm{e}}$ et XII ${ }^{\mathrm{e}}$ siècles, se manifeste à travers la quête de la solitude, d'après les modèles des Pères du Désert, associés à la pastorale apostolique, le tout dans le cadre d'une Église de plus en plus « étatique ». Le silence comme pratique politique reste encore un champ en friche pour les médiévistes. 